\title{
Discours
}

Revue de linguistique, psycholinguistique et

informatique. A journal of linguistics, psycholinguistics

and computational linguistics

$2 \mid 2008$

Varia

\section{Les verbes de parole et la question de l'(in)transitivité}

Béatrice Lamiroy et Michel Charolles

\section{OpenEdition}

Journals

Édition électronique

URL : http://journals.openedition.org/discours/3232

DOI : $10.4000 /$ discours.3232

ISSN : 1963-1723

Éditeur :

Laboratoire LATTICE, Presses universitaires de Caen

Référence électronique

Béatrice Lamiroy et Michel Charolles, «Les verbes de parole et la question de l'(in)transitivité »,

Discours [En ligne], 2 | 2008, mis en ligne le 11 octobre 2017, consulté le 19 avril 2019. URL : http:// journals.openedition.org/discours/3232 ; DOI : 10.4000/discours.3232

Ce document a été généré automatiquement le 19 avril 2019

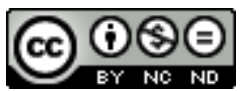

Discours est mis à disposition selon les termes de la licence Creative Commons Attribution - Pas d'Utilisation Commerciale - Pas de Modification 4.0 International. 


\title{
Les verbes de parole et la question de l'(in)transitivité
}

\author{
Béatrice Lamiroy et Michel Charolles
}

\section{Introduction}

1 La problématique explorée dans cet article concerne la question de l'articulation entre la transitivité syntaxique et la sémantique verbale. Si on prend un champ sémantique a priori relativement homogène ${ }^{1}$ comme celui des verbes de parole, la question qui se pose est de savoir pourquoi par exemple dire est transitif, parler est transitif indirect, alors que digresser est intransitif :

[1a] Paul dit que Marie lui manque.

[1b] Paul parle de Marie.

[1c] ?* Paul digresse (que Marie lui manque + de Marie).

2 L'hypothèse que nous avions adoptée dans une première exploration de ces verbes (Charolles, Lamiroy, 2001) est que la distribution entre verbes transitifs et intransitifs n'est pas totalement aléatoire, qu'elle est déterminée au contraire par le champ sémantique particulier dont les verbes font partie. Ainsi, les verbes de mouvement seraient de façon prédominante de nature intransitive, parce qu'ils servent essentiellement à localiser une entité par rapport à l'espace (Sarda, 2000) ${ }^{2}$. Les verbes de parole, en revanche, incluraient dans leur représentation conceptuelle profonde un objet, à savoir les paroles produites et, dans la mesure où ils impliquent par nature un objet, ils seraient naturellement transitifs.

Un fait qui vient corroborer cette hypothèse est qu'on trouve avec les verbes de parole toutes les propriétés typiques associées à la transitivité : celle-ci se caractérise par une asymétrie fondamentale entre deux participants dont l'un, typiquement humain, exerce intentionnellement une action télique sur un objet typiquement inanimé, effectué ou affecté (Herslund, 2001; Hopper, Thomson, 1985; Lazard, 1994; Levin, 1993; Talmy, 1985). En effet, prototypiquement, le sujet d'un verbe de parole est de type $\mathrm{N}$ [+humain], 
alors que l'objet est au contraire inanimé. Par ailleurs, la production de paroles est en général - sauf exception, dans des cas comme délirer - une activité contrôlée par un sujet agentif, qui renvoie à un processus télique, puisqu'elle a la production langagière pour objet.

\section{Les verbes de parole transitifs et intransitifs: Charolles et Lamiroy (2001)}

\subsection{Définitions}

4 L'étude quantitative de (Charolles, Lamiroy, 2001) est fondée sur une liste de 515 verbes sélectionnés sur la base d'un critère sémantique : les verbes de parole prennent un sujet humain et ne peuvent entrer dans une construction du type $\mathrm{N}_{0} \mathrm{~V}\left(\mathrm{E}+(\mathrm{de}+\mathrm{E}) \mathrm{N}_{1}\right)+((\mathrm{de}$ ce + E) Que P) ) $)^{3}$, sans que $\mathrm{N}_{0}$ dise ou écrive un mot.

D’après Gross (1975 : table 9), ce genre de verbes se définit par la structure suivante :

[2a] No V (N1 + que P) à N2

[2b] Paul annonce l'arrivée de Marie à Pierre.

[2c] Paul annonce que Marie arrive à Pierre.

Giry-Schneider (1981 : 1994) note une deuxième structure pour des verbes tels que prier, défier, avertir, accuser, menacer, remercier, convaincre, féliciter, instruire:

[3a] No V N1 de N2

[3b] Paul avertit Pierre de l'arrivée de Marie.

La structure intransitive peut être représentée comme suit :

[4a] No V (E+ NLoc)

[4b] Paul (zozote + bégaye + tchatche) au bureau.

Du point de vue sémantique, un verbe de parole est un verbe qui dénote une activité linguistique du sujet parlant visant normalement ${ }^{4}$ à communiquer un message à quelqu'un. Comme il y a transmission d'un « objet » (le message) à un tiers, les verbes de parole ont été mis en rapport avec les verbes datifs (Guillet, Leclère, 1992 ; Kübler, 1994).

9 Les verbes de parole retenus dans (Charolles, Lamiroy, 2001) ont été annotés comme transitifs ou intransitifs à partir de dictionnaires (Robert, Bescherelle) et ont été complétés par un examen des tables 6 (ex. revendiquer) et 9 (ex. dire) de Gross (1975), des tables 31H (ex. cabotiner), 34Lo (ex. aboyer) et 35R (ex. blaguer) de Boons et al. (1976) et des tables 36 DT (ex. chicaner), 37 M1 (ex. argumenter) et 38Lo (ex. éructer) de (Guillet, Leclère, 1992). La répartition - 149 verbes intransitifs versus 366 transitifs - fait apparaitre un net avantage en faveur des transitifs, avantage qui va globalement dans le sens de l'hypothèse que les verbes de parole sont tendanciellement transitifs.

\subsection{Principe de saturation sémantique}

10 Puisque les verbes de parole sont, majoritairement, des verbes transitifs, la question se pose de savoir pourquoi certains verbes de parole sont tout de même intransitifs. L'hypothèse explorée dans (Charolles, Lamiroy, 2001) et (Lamiroy, 2005) propose, comme 
une des explications possibles, de relier l'intransitivité des verbes de parole à un principe de saturation sémantique ${ }^{5}$. Celui-ci prédit un rapport inverse entre transitivité syntaxique et spécification sémantique: plus le sémantisme d'un verbe serait spécifié, plus le verbe serait saturé lexicalement et plus il serait intransitif. Ainsi, plus le verbe spécifie par exemple la manière de parler, plus il aurait tendance à ne pas profiler l'objet. C'est ce qui est notamment suggéré par les paires suivantes:

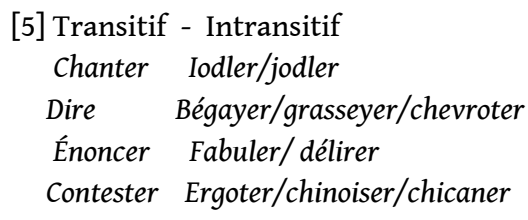

\subsection{Transitivité et aspect}

11 La plupart des travaux consacrés à la question de la transitivité (François, 1999; Givon, 1984 ; Langacker, 1987) ont mis en évidence qu'il existe un rapport intrinsèque entre intransitivité et atélicité du procès exprimé, ce lien étant le pendant de la relation prototypique entre transitivité et télicité. ${ }^{6}$ La deuxième hypothèse que nous avons adoptée est donc la suivante: lorsque le sémantisme du verbe dénote une activité atélique, ce qui est saillant est le déroulement même de l'activité de parole qu'indique le verbe plutôt que son objet. Par conséquent, le verbe sera par défaut intransitif. En effet, l'objet (effectué ou affecté) n'est pas profilé, et donc pas facilement récupérable. C'est ce qui semble expliquer l'opposition entre des paires comme celles de [6] :

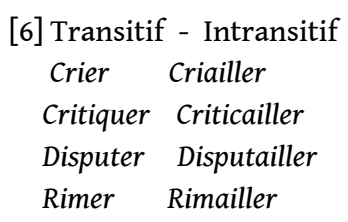

Les quelques exemples donnés ci-dessous montrent que les deux principes que nous venons d'indiquer ne valent apparemment pas uniquement pour les verbes de parole :

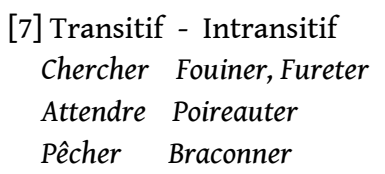

\subsection{Classement sémantique des verbes de parole}

13 Pour affiner cette double hypothèse et voir quels facteurs pouvaient favoriser l'intransitivité, nous avons entrepris dans (Charolles, Lamiroy, 2001) un premier classement de l'ensemble des verbes de parole retenus en fonction d'un certain nombre ${ }^{7}$ de critères sémantiques ${ }^{8}$, à savoir :

- (1) la source des paroles : verbes comme réciproquer ou commenter qui impliquent qu'il y ait eu des paroles antérieures ;

- (2) la forme du message : verbes comme zézayer ou murmurer qui indiquent la façon dont les paroles sont proférées ; 
- (3) le caractère non programmé du message: verbes tels que papoter ou bavarder qui indiquent que le contenu des paroles ${ }^{9}$ n'est pas programmé au préalable ;

- (4) le locuteur: verbes qui spécifient soit l'état mental du locuteur (ex. délirer) soit une attitude psychologique (ex. fulminer) ;

- (5) l'acte de langage : verbes dénotant différents types d'actes de langage tels qu'ils ont été établis par Searle $(1979,1982)$ tels que décréter (acte déclaratif), (dé)conseiller (acte directif), implorer (acte assertif) et féliciter (acte expressif) ;

- (6) le caractère générique du verbe : verbes neutres tels que parler ou dire.

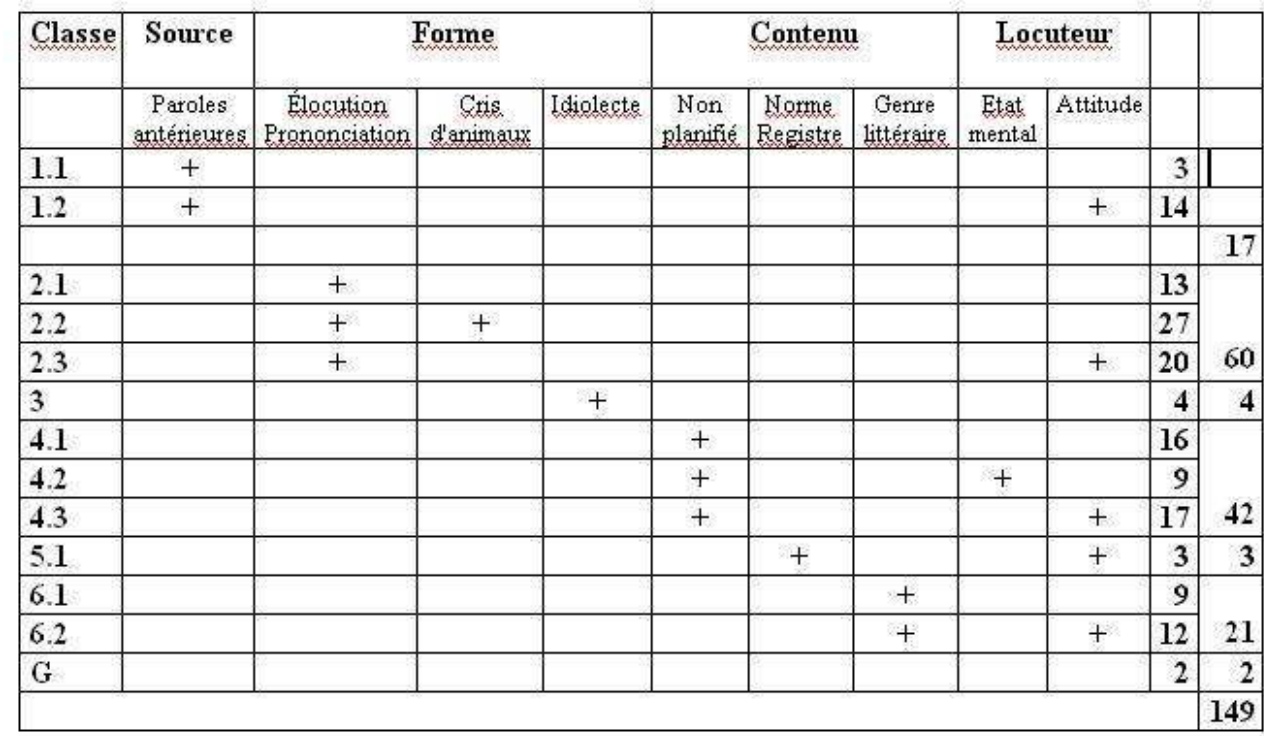

Tableau 1 : Classes sémantiques de verbes de parole

Classe VI.1.1 : Réciproquer, Recauser, Reparler

Classe VI.1.2: Acquiescer, Broder, Chicaner, Chinoiser, Chipoter, Convenir, Digresser, Disconvenir, Ergoter, Insister, Mégoter, Pinailler, Polémiquer, Tergiverser

Classe VI.2.1: Babiller, Bafouiller, Bégayer, Bléser, Chevroter, Couiner, Criailler, Iodler, Jodler, Grasseyer, Nasiller, Zézayer, Zozoter

Classe VI.2.2: Aboyer, Barrir, Bêler, Beugler, Blatérer, Braire, Bramer, Cacarder, Caqueter, C(r)oasser, Feuler, Gazouiller, Glapir, Glousser, Hennir, (H)ululer, Jacasser, Japper, Meugler, Miauler, Mugir, Pépier, Piailler, Piauler, Ronronner, Roucouler, Striduler

Classe VI.2.3 : Cafouiller, Éructer, Fanfaronner, Fulminer, Grognasser, Grognonner, Jaboter, Maugréer, Pester, Protester, Rager, Râler, Récriminer, Rouscailler, Rouspéter, S'esclaffer, Tempêter, Tonitruer, Tonner, Vitupérer

Classe VI. 3 : Jargonner, Joualiser, Jurer ('utiliser des jurons), Patoiser

Classe VI.4.1: Bavarder, Blablater, Converser, Deviser, Discourir, Discutailler, Discuter, Ecrivailler, Ecrivasser, Jacter, Jaspiner, Papoter, Parloter, Pérorer, Tartiner, Tchatcher

Classe VI.4.2: Délirer, Dérailler, Déraisonner, Divaguer, Extravaguer, Fabuler, Radoter, Ratiociner, Vaticiner

Classe VI.4.3: Bavasser, Baver, Bavocher, Blaguer, Cancaner, Clabauder, Criticailler, Déblatérer, Dégoiser, Galéger, Ironiser, Jaser, Médire, Plaisanter, Potiner, Soliloquer, Targuer(se)

Classe VI. 5.1 : Mentir, Apostasier, Parjurer

Classe VI.6.1 : Argumenter, Conférer, Débattre, Disputer, Disserter, Laïusser, Plancher, Pétrarquiser, Rimailler

Classe VI.6.2: Baratiner, Bonimenter, Cabotiner, Dogmatiser, Disputailler, 
épiloguer, Marivauder, Motionner, Palabrer, Parlementer, Pétitionner, Pontifier

Classe VI.G : Parler, Causer

\section{sont transitifs, les verbes se répartissent dans les deux classes, transitive et intransitive} respectivement. Les chiffres sont reproduits dans le tableau ci-dessous :

\begin{tabular}{|l|l|l|}
\hline & V intransitifs & V transitifs \\
\hline 1. Source des paroles & $11 \%$ & $39 \%$ \\
\hline 2. Forme des paroles & $42 \%$ & $30 \%$ \\
\hline 3. Contenu des paroles & $44 \%$ & $9 \%$ \\
\hline 4. Locuteur & $50 \%$ & $17 \%$ \\
\hline 5. Acte de langage & $0 \%$ & $61 \%$ \\
\hline 6. Verbe générique & $2 \%$ & $4 \%$ \\
\hline
\end{tabular}

Tableau 2 : Répartition des verbes de parole en types sémantiques

\subsection{Principaux résultats de Charolles et Lamiroy (2001)}

Le classement donné ci-dessus appelle un certain nombre de commentaires, qui correspondent aux résultats les plus saillants de (Charolles, Lamiroy, 2001) :

- Pour ce qui est de l'élément déclencheur de la production verbale: $11 \%$ des verbes intransitifs spécifient ce trait, contre $39 \%$ des verbes transitifs. Les verbes intransitifs sont donc nettement moins nombreux que les verbes transitifs à présupposer une source, un discours déclencheur.

- La spécification de la forme favorise l'intransitivité du verbe, mais ce n'est pas une règle absolue, puisque certains verbes transitifs caractérisent aussi parfois la production de la parole. En effet, des verbes comme marmonner, murmurer, grommeler ou bredouiller indiquent que la production de paroles se fait, respectivement, à voix basse, avec une certaine hostilité ou de façon peu claire. Et pourtant il s'agit bien de verbes transitifs. Toutefois, les verbes intransitifs sont bien plus nombreux (p. ex. babiller, bégayer, chevroter, couiner, criailler, grasseyer, iodler, joualiser, laïsser, nasiller, zézayer, zozoter).

- Lorsqu'un verbe spécifie d'une façon ou d'une autre le contenu de la production de paroles, le contraste est net: aucun verbe transitif ne dénote une activité de parole non planifiée, atélique et par défaut durative, alors que c'est le cas pour près d'un tiers des verbes intransitifs, par ex. bavarder, parloter, pérorer, tartiner. De même, une proportion beaucoup plus importante de verbes intransitifs marque la conformité à une norme, un registre ou un genre de discours, p. ex. chinoiser, pindariser. Les verbes transitifs sont nombreux en revanche (22 verbes) à indiquer une transformation du contenu (p. ex. abréger), catégorie qui, comme on pouvait s'y attendre, n'est représentée par aucun verbe intransitif: l'objet affecté est (avec l'objet effectué, après un verbe comme construire par exemple) un des objets types des verbes transitifs.

- Les verbes intransitifs caractérisant l'attitude du locuteur sont trois fois plus nombreux que les transitifs. Les verbes de parole intransitifs se prêtent ainsi particulièrement à décrire l'état mental ou psychologique du locuteur: ils caractérisent l'activité langagière en fonction des dispositions intellectuelles du sujet parlant.

- Pour ce qui est du marquage de la valeur d'acte de langage, la différence entre les intransitifs et les transitifs est très nette puisque aucun verbe intransitif n'exprime un acte de langage, alors que 226 verbes transitifs $(61 \%$ des $\mathrm{Vt})$ satisfont à ce trait. 
- Les verbes de parole appelés "génériques" (sous-spécifiés sémantiquement) sont relativement peu nombreux dans les deux catégories, avec seulement 2 ou 3 verbes intransitifs génériques (causer, parler, plus, si on veut, tchatcher) et une dizaine de verbes transitifs (répartir, répondre, répliquer, déclarer, dire, émettre, énoncer, exposer, exprimer, formuler, écrire, libeller, rédiger). Le fait qu'il y ait nettement moins de verbes de parole génériques intransitifs que transitifs est conforme à l'idée que les verbes de parole sont par défaut plutôt transitifs de nature.

Ces résultats montrent que les hypothèses émises au départ de cette première étude ne sont pas fausses mais que leur validité doit être relativisée, dans la mesure où la plupart des verbes de parole se répartissent dans les deux ensembles, dans des proportions diverses. Pour les verbes de parole comme pour les verbes en général (Hopper, Thomson, 1985), nous souscrivons à un principe de gradation scalaire prévoyant un continuum allant de verbes prototypiquement transitifs à des verbes prototypiquement intransitifs avec, logiquement, une série de cas intermédiaires. Ceux-ci correspondent essentiellement à trois cas de figure : soit les verbes sont à la fois transitifs et intransitifs [8], soit ils sont foncièrement intransitifs mais peuvent être occasionnellement transitifs ${ }^{10}$ [9-10], soit ils sont transitifs [11-12] mais s'emploient facilement de manière absolue (Larjavaara, 2000) :

[8a] A partir d'un certain âge on radote.

[8b] Qu'est-ce qu'il radote?

[9a] Elles parlent (de chiffons + chiffons).

[9b] Elle cause (en anglais + anglais).

[10a] Paul adore blaguer.

[10b] Ses copains l'appelaient, lui racontaient des histoires, le blaguaient. (R.

Sabatier, Les fillettes chantantes, 1980)

[11a] Paul me tutoie depuis longtemps.

[11b] En Espagne on tutoie volontiers.

[12] Cette jolie demoiselle plutôt discrète, qui déteste l'impudeur mais s'avoue volontiers "voyeuse", ne claironne pas sur les toits, [...]. Et pourtant, elle a tout signé : dessin de l'affiche, costume, mise en scène. (ex. de Larjavaara, $2000: 70$ )

Dans une situation où il y a des passages possibles d'une structure à l'autre, une étude de corpus devrait permettre de nuancer les choses en précisant laquelle des deux structures est dominante par rapport à l'autre. La suite de cet article est consacrée à la présentation d'une telle étude, à partir des verbes de parole étiquetés comme intransitifs dans le Petit Robert électronique (1997).

\section{Etude sur corpus des verbes de parole intransitifs}

Les verbes retenus pour l'étude sur corpus dont les résultats sont présentés ci-après ont été sélectionnés dans le Petit Robert électronique (PRe) sur la base de la formule présentée sous 1. Pour chacun des verbes considérés, nous avons recueilli dans Frantext (version catégorisée) au maximum 100 exemples d'emplois dans les essais écrits entre 1950 et $2000^{11}$. Lorsque cette première interrogation ne donnait pas le nombre d'emplois attendu, la sélection a été étendue aux romans parus entre 1950 et 2000, et si besoin à la base entière. Seuls ont été retenus les emplois dans lesquels le sujet est un animé, ce qui 
exclut les emplois métaphoriques du type le train beugle. Nous avons également éliminé tous les verbes pronominaux (p. ex. s'exclaffer, se targuer) ${ }^{12}$.

Les emplois recueillis sont parfois peu nombreux (par ex. grognasser : 2 cas; iodler : 1 cas), voire inexistants (par ex. jodler, réciproquer et tchatcher) et donc non pris en compte dans la suite. La liste des 142 verbes de parole pour lesquels nous avons recueillis au moins un emploi dans Frantext s'établit comme suit ${ }^{13}$.

\begin{tabular}{|c|c|c|c|c|c|}
\hline Aboyer & Acquiescer & Apostasier & Argumenter & Babiller & Bafouiller \\
\hline Baratiner & Barrir & Bavarder & Bavasser & Baver & Bavocher \\
\hline Bégayer & Bêler & Beugler & Blablater & Blaguer & Blatérer \\
\hline Bléser & Bonimenter & Braire & Bramer & Broder & Cabotiner \\
\hline Cacarder & Cafouiller & Cancaner & Caqueter & Causer & Chevroter \\
\hline Chicaner & Chinoiser & Chipoter & Clabauder & Coasser & Conférer \\
\hline Convenir & Converser & Couiner & Criailler & Criticailler & Croasser \\
\hline Débattre & Déblatérer & Dégoiser & Délirer & Dérailler & Déraisonner \\
\hline Deviser & Digresser & Disconvenir & Discourir & Discutailler & Discuter \\
\hline Disputailler & Disputer & Disserter & Divaguer & Dogmatiser & Ecrivailler \\
\hline Ecrivasser & Epiloguer & Ergoter & Eructer & Extravaguer & Fabuler \\
\hline Fanfaronner & Feuler & Fulminer & Galéjer & Gazouiller & Glapir \\
\hline Glousser & Grasseyer & Grognasser & Grognonner & Hululer & Insister \\
\hline Lodler & Ironiser & Jaboter & Jacasser & Jacter & Japper \\
\hline Jargonner & Jaser & Jaspiner & Jurer & Laüusser & Marivauder \\
\hline Maugréer & Médire & Mentir & Meugler & Miauler & Motionner \\
\hline Mugir & Nasiller & Opiner & Palabrer & Papoter & Parjurer \\
\hline Parlementer & Parler & Parlater & Patoiser & Pépier & Pérorer \\
\hline Pester & Pétitionner & Pétrarquiser & Piauler & Pinailler & Pindariser \\
\hline Plaisanter & Plancher & Polémiquer & Pontifier & Potiner & Protester \\
\hline Radoter & Rager & Raler & Ratiociner & Recauser & Récriminer \\
\hline Reparler & Rimailler & Ronronner & Roucouler & Rouscailler & Rouspéter \\
\hline Soliloquer & Striduler & Tartiner & Tempêter & Tergiverser & Tonitruer \\
\hline Vaticiner & Vitupérer & Zézayer & Zozoter & & \\
\hline
\end{tabular}

Tableau 3 : Liste des 142 verbes de parole intransitifs attestés dans Frantext

20 Avant de discuter des emplois auxquels ces verbes peuvent se prêter, il est important de souligner que, pour le décompte des constructions, nous avons noté comme transitifs les emplois dans lesquels le verbe est suivi d'un discours direct ou figure en incise. Bien qu'il ne s'agisse pas de c.o.d orthodoxes, ces constituants peuvent être analysés comme des compléments d'objet pour plusieurs raisons. Premièrement, ils répondent à la question en qu'est-ce que, pronom interrogatif qui renvoie au complément d'objet direct:

[13a] Qu'est ce que Paul a dit?

[13b] Il a dit qu'il va partir à la campagne.

[13c] Paul a dit : je vais partir à la campagne.

[13d] Je vais partir à la campagne, a-t-il dit.

Deuxièmement, on ne peut ajouter au verbe en incise un autre complément d'objet :

[14a] Comprends-moi bien, dit Jacques, je ne refuse pas de te rendre service. (Sartre, cité par Riegel 1994 : 461)

[14b] * Comprends-moi bien, dit Jacques une chose, je ne refuse pas de te rendre service.

Ces tests très simples montrent que les paroles proférées correspondent au complément du verbe de parole. En discours direct, on peut bien sûr ajouter un c.o.d. après un verbe de parole tel que dire, mais les paroles rapportées devront, dans ce cas, être analysées comme une apposition au c.o.d. Autrement dit, si on peut attribuer au discours direct ou à la phrase suivie d'une incise une fonction, cela ne peut être que celle de c.o.d. (ou 
apposition à celui-ci). Riegel (1994 : 460) analyse l'incise comme un cas d'insertion, mais précise en note que «l'incise correspond à la phrase introductive qui est placée avant une séquence de discours indirect: la transposition de l'exemple de Sartre peut aboutir à Jacques dit qu'il ne refusait pas de lui rendre service». Dans cette dernière phrase, la complétive qu'il ne refusait ... est évidemment le complément d'objet direct de dit, ce qui confirme tout à fait notre analyse.

Une telle approche n'est possible que si on souscrit au principe de gradation auquel nous avons fait allusion dans la partie précédente et sur lequel nous reviendrons plus loin : si la transitivité stricte est représentée par une structure du type No $V N 1$, le genre de construction auquel nous avons affaire ici représente une transitivité faible. Transitivité faible, mais transitivité tout de même. Nous proposons l'échelle suivante, allant du cas le moins transitif à celui qui l'est le plus :

incise $<$ discours direct $<\mathrm{SN}<$ que $\mathrm{P}<$ clitique

Figure 1 : Échelle de transitivité de compléments de type nominal : le verbe croasser apparaît 17 fois en emploi transitif dans le corpus dont 5 correspondent à des incises [16a], 3 à du discours direct [16b], alors qu'il n'y a que deux cas de SN [16c] :

[16a] «Ce vieux cochon d'Abdoul Hamid ! Satyre libidineux ! " croassait-il, surtout en présence de mes serviteurs, et je frissonnais de peur à l'idée que l'un d'eux comprît le français. (M. de Grèce, La nuit du sérail, 1982)

[16b] Je vis mon ami, l'officier français, sommeillant en paix, tandis qu'un grand corbeau planait au-dessus de sa tête en croassant : crââ, crââ, l'ombre des gommiers de Gâo-crââ, crââ, ne vaudra rien la nuit prochaine- crââ, crââ, au chef blanc, ni à son escorte. (P. Benoit, L'Atlantide, 1919)

[16c] Le troubadour de la guillotine, sur le rapport duquel la convention décréta que la terreur était à l'ordre du jour, échappa à cette terreur en se cachant dans le panier aux têtes; $d u$ fond du baquet de sang, sous l'échafaud, on l'entendait seulement croasser la mort ! (F. de Chateaubriand, Mémoires d'outre-tombe, 1848)

26

\begin{abstract}
[15a] "Elle est à vous, la grosse voiture américaine devant le journal ? "
cafouilla-t-elle, deux tons plus bas. (M. Embareck, Sur la ligne blanche, 1984) [15b] « Oh, dis donc », feula-t-elle, « laisse-moi respirer, tu veux ? Je suis pas en cavale, moi ! » (J. Vautrin, Bloody Mary, 1979)
$[15 \mathrm{c}]$ « On peut être amoureux tout un jour de Mme Tallien », médisent les

uns. "Elle n'inspire que les plaisirs des sens", renchérissent les autres. Kristeva, Les samouraïs, 1990)
\end{abstract} de complément des cas d'incise : c'est le cas, dans le corpus étudié, de verbes comme par exemple cafouiller [15a], feuler [15b] ou médire [15c]: accompagnement de paroles rapportées [17a], mais dans 4 cas seulement le verbe est suivi d'une complétive [17b] et dans 2 cas par un SN [17c] :

[17a] On en fait tout un plat du Jésus parce qu'il s'est rétamé trois fois, maugréait Juju, moi j'en prends un peu plus, des pelles, et il avait pas une 
musette et un radiateur à se coltiner, le frère. (R. Fallet, La grande ceinture, 1956)

[17b] En outre, lorsque vous avez la chance de réagir au printemps sur la route sans maugréer qu'il s'agit là du temps rêvé pour les averses, le rhume des foins, les coups de soleil, les moustiques, la malaria et le scorbut floral, c'est qu'il vous reste un coeur, des yeux pour voir, un zeste d'ange. (R. Fallet, Le triporteur, 1951)

[17c] Elle maugréa les pires injures à son intention, le traita de jean-foutre, de ruchemerde, de cornecul et de godebillard, de propoune et de bandouillette. (J. Lanzmann, La Horde d'Or, 1994)

Bien que les verbes qui admettent une complétive admettent le plus souvent un SN, il s'avère que les syntagmes nominaux sont en fait bien plus courants dans le corpus que les complétives : pour bafouiller, p. ex. on trouve trois cas de complétive [18a], contre vingtcinq cas de SN [18b], pour baratiner deux cas de que $P$ contre dix-sept cas de SN :

[18a] Je bafouille que ça doit être un courant d'air : avec cette porte toujours ouverte, vous comprenez? (J.-L. Benoziglio, Cabinet portrait, 1980) [18b] Nadine se mit à rire : Lambert a eu l'air soufflé, il a bafouillé des choses mais je ne l'ai pas laissé parler. (S. de Beauvoir, Les mandarins, 1954)

Quant à la pronominalisation du $\mathrm{SN}$, on observe que les verbes qui prennent un $\mathrm{SN}$ n'admettent pas automatiquement un pronom :

[19a] Il était alors cinq heures du matin. Un oiseau vint s'abattre sur les pots de fleurs qui se trouvaient en dehors de la croisée, et gazouilla quelques chants. (H. de Balzac, Illusions perdues 1843)

[19b] ? ? Un oiseau vint s'abattre ... et en gazouilla quelques-uns.

\begin{tabular}{|c|c|c|}
\hline $\begin{array}{c}\text { Emplois } \\
\text { intransitifs à }\end{array}$ & $\begin{array}{l}142 \text { verbes intransitifs } \\
\text { attestés dans Frantext }\end{array}$ & $\begin{array}{c}\% \\
\text { (effectif) }\end{array}$ \\
\hline $100 \%$ & $\begin{array}{l}\text { acquiescer ; aapostasier ; babiller ; bavarder ; bavocher ; blablater ; } \\
\text { blatérer ; bléser ; cabotiner ; converser ; digresser ; discutailler ; } \\
\text { disputailler ; disserter ; divaguer ; écrivasser ; galéjer ; iodler ; } \\
\text { laüusser; marivauder ; mentir ; motionner ; parlementer ; parloter ; } \\
\text { pétrarquiser ; pinailler ; pindariser ; plancher ; recauser ; reparler : } \\
\text { striduler ; tergiverser }\end{array}$ & $\begin{array}{l}22,5 \% \\
(32 \mathrm{~V})\end{array}$ \\
\hline $90-100 \%$ & $\begin{array}{l}\text { bavasser ; braire ; cancaner ; causer ; coasser ; conférer ; criailler; } \\
\text { déblatérer ; délirer ; dérailler ; déraisonner ; deviser ; discourir ; } \\
\text { épiloguer ; ergoter ; extravaguer ; feuler; hululer ; insister ; jaboter } \\
\text { jacasser ; japper ; jaser ; médire ; meugler ; palabrer ; papoter ; } \\
\text { parjurer ; parler ; pépier ; pester ; piauler ; polémiquer ; pontifier ; } \\
\text { potiner ; récriminer ; ronronner }\end{array}$ & $\begin{array}{l}26 \% \\
(37 \mathrm{~V})\end{array}$ \\
\hline $80-90 \%$ & $\begin{array}{l}\text { aboyer ; barrir ; bêler ; cacarder ; cafouiller ; caqueter ; chipoter : } \\
\text { clabauder ; croasser ; dogmatiser ; écrivailler ; fabuler ; fanfaronner } \\
\text { miauler; mugir ; pérorer ; protester ; radoter ; ratiociner ; tempéter ; } \\
\text { zézayer ; zozoter }\end{array}$ & $\begin{array}{l}15,5 \% \\
(22 \mathrm{~V})\end{array}$ \\
\hline $70-80 \%$ & $\begin{array}{l}\text { blaguer ; broder ; cotiner ; disconvenir : gazouiller ; grognonner; } \\
\text { jacter ; patoiser ; pétitionner ; plaisanter ; rimailler ; rouspéter ; } \\
\text { soliloquer }\end{array}$ & $\begin{array}{c}9 \% \\
(13 \mathrm{~V})\end{array}$ \\
\hline $60-70 \%$ & $\begin{array}{l}\text { bonimenter : bramer ; glousser ; jargonner : jaspiner ; maugréer; } \\
\text { opiner ; räler; roucouler ; vaticiner }\end{array}$ & $\begin{array}{l}7 \% \\
(10 \mathrm{~V})\end{array}$ \\
\hline $50-60 \%$ & $\begin{array}{l}\text { argumenter ; baver ; bégayer ; chevroter ; éructer; fulminer ; grasseyer } \\
\text {; rouscailler; tartiner ; tonitruer }\end{array}$ & $\begin{array}{l}7 \% \\
(10 \mathrm{~V})\end{array}$ \\
\hline $40-50 \%$ & bafouiller; ironiser, rager: & $\begin{array}{l}2 \% \\
(3 \mathrm{~V})\end{array}$ \\
\hline $30-40 \%$ & beugler ; chicaner ; chinoiser ; débattre ; discuter ; nasiller & $\begin{array}{l}4 \% \\
(6 \mathrm{~V})\end{array}$ \\
\hline $20-30 \%$ & convenir ; dégoiser ; jurer; vitupérer ; glapir & $\begin{array}{l}3 \% \\
(5)\end{array}$ \\
\hline $10-20 \%$ & baratiner : disputer & $\begin{array}{c}1,5 \% \\
(2)\end{array}$ \\
\hline $0-10 \%$ & criticailler; grognasser & $\begin{array}{c}1,5 \% \\
(2)\end{array}$ \\
\hline
\end{tabular}

Tableau 4 : Emplois (in)transitifs des 142 verbes de parole dans Frantext

Avant d'examiner de plus près les données recueillies, on relèvera au plan général que beaucoup de verbes étiquetés comme intransitifs par le PRe ont des emplois transitifs attestés, ce qui tend à confirmer notre hypothèse initiale selon laquelle les verbes de 
parole sont de nature transitifs. Par ailleurs $22,5 \%$ des verbes étiquetés dans le PRe comme intransitifs ne sont jamais attestés dans le corpus de Frantext en emploi transitif. $\mathrm{Si}$ on y ajoute les verbes dont le pourcentage d'exemples intransitifs attestés dans Frantext se situe entre 80 et $100 \%$ des cas, on arrive à un taux de $64 \%$ du total. Ces verbes peuvent être considérés comme les intransitifs "durs ». Mais on observe également qu'une partie non négligeable de verbes sont transitifs dans la moitié ou plus de leurs emplois : c'est le cas de 18 verbes, ce qui correspond à $12,5 \%$ du total. Si on y ajoute ceux de la catégorie supérieure, à savoir ceux qui sont transitifs dans 40 à $50 \%$ des cas, on arrive à 28 verbes, soit $20 \%$ de l'ensemble. Il faut toutefois souligner que, même si ces fluctuations entre structures transitives et intransitives sont incontestables, la plupart des verbes ont, malgré tout, un emploi préférentiel. Autrement dit, certains verbes résistent à un passage éventuel d'intransitif à transitif. On comparera à cet égard les exemples $[20 \mathrm{a}-\mathrm{b}-\mathrm{c}]$ :

[20a] Ils sont capables de (lä̈usser + joualiser) pendant des heures.

[20b] ? Ils ont (lä̈ussé + joualisé) de leur travail.

[20c] ?* Ils ont (laïussé + joualisé) que les verbes de parole sont intéressants.

Pour les verbes dont Frantext fournit des attestations d'emplois transitifs et intransitifs à la fois, nous allons examiner maintenant de plus près quels procédés permettent le passage d'une structure à l'autre, en commençant par le noyau dur des verbes intransitifs.

\subsection{Verbes de parole intransitifs « durs »}

31 Nous avons regroupé sous cette catégorie les verbes qui présentent 100 à $80 \%$ d'attestations d'emplois intransitifs. On y retrouve les catégories sémantiques caractérisées dans la partie précédente comme typiques des emplois intransitifs, notamment les verbes :

- indiquant la façon dont les paroles sont produites : babiller, zézayer, zozoter ainsi que ceux renvoyant à des cris d'animaux: aboyer, barrir, bêler, blatérer, braire, cancaner, clabauder, coasser, caqueter, feuler, croasser, hululer, hennir, meugler, pépier, piauler, ronronner ;

- dénotant une activité de type atélique : criailler, discutailler, disputailler, écrivailler, écrivasser ${ }^{14}$;

- renvoyant à l'état mental du locuteur: divaguer, digresser, délirer, extravaguer, dérailler, déraisonner, fabuler, radoter, ratiociner ;

- renvoyant au contenu non planifié des paroles : bavarder, bavasser, blablater, causer, conférer, converser, discourir, deviser, galéjer ; parloter, potiner ;

En voici quelques exemples :

[21a] Je m'épiais : une heure plus tôt j'avais babillé avec ma mère : qu'avaisje annoncé? (J.-P. Sartre, Les mots, 1964)

[21b] Ils discutaillent dans un jargon imbitable pour moi. (E. Hanska, Les amants foudroyés, 1984)

[21c] Il avait construit tout le drame et les propos confus d'un fiévreux, qui lui-même délirait, n'avaient pu lui rendre son bon sens. Toujours la même folie de pauvre. (J. Guéhenno, Jean-Jacques, 1952)

[21d] La petite ? Allez, vaï, vous galéjez ! (M. Pagnol, Marius, 1931)

Rappelons que, comme nous l'avons signalé plus haut, aucun verbe indiquant un acte de langage, n'a été trouvé en emploi intransitif. Un fait important qui saute aux yeux quand 
on examine les verbes ci-dessus est que ceux qui renvoient à des cris d'animaux sont particulièrement nombreux. Or ils occupent une place à part parmi les verbes intransitifs « durs » dans la mesure où l'emploi intransitif renvoie au sens premier de ces verbes. En effet, l'emploi transitif est courant pour ces verbes ${ }^{15}$ lorsqu'ils adoptent un sens métaphorique. On voit bien cette spécialisation fonctionnelle avec hululer qui est toujours intransitif quand il est employé au sens propre ([22a]) et le plus souvent transitif (mais pas toujours ! voir [22d]) quand il a un sens métaphorique ([22b] et [22c]) :

[22a] Il resta encore un certain temps à rêver en fumant des cigarettes et en buvant des petits verres. De temps à autre, un oiseau de nuit hululait. Il en élevait pour son plaisir. Il ne cherchait pas à les dresser. Il connaissait la voix de chacun d'eux. (R. Queneau, Pierrot mon ami, 1942)

[22b] La traversée fut brève ; accroupi dans un coin de la cabine de poupe, Euphorion hululait à voix basse je ne sais quelle complainte funèbre africaine ; ce chant étouffé et rauque me semblait presque mon propre cri. Nous transférâmes le mort dans une salle lavée. (M. Yourcenar, Mémoires d'Hadrien, 1951)

[22c] « Grande fête! » hululait toujours Michel. «Qui aura son canari? Je dis bien son canari-ri-ri! » (H. Bazin, Le bureau des mariages, 1951)

[22d] Elle grimpe jusqu'aux plates-formes où les femmes et les filles hululent de joie à chaque coup de feu qui ne frappe pas leurs proches; sa face exsangue entre ses longues nattes noires prend place sur les créneaux dans la file des têtes tranchées. (M. Yourcenar, Feux. Antigone ou le choix, 1936) (exemples [a] ci-dessous) d'une part, et entre emplois intransitifs et sens littéraux d'autre part (exemples [b] ci-dessous) dans l'ensemble du corpus :

[23a] Quand donc s'arrêtera-t-il, cet air bêlé par une voix stupide... (c. Mauriac, La marquise sortit à cinq heures, 1961)

[23b] On riait d'eux en disant : «Voilà des moutons pressés de se faire tondre et écorcher, ils courent au-devant du boucher et bêlent de joie. " (Oldenbourg, Z., Les cités charnelles ou l'histoire de Roger de Montbrun, 1961)

[24a] Enfin, je croyais ce mot-là réservé au dictionnaire des midinettes. A le bramer dans les courriers du cœur, on l'a dévalorisé. (H. Bazin, La mort du petit cheval, 1950)

[24b] Comme le cerf, selon le psalmiste, brame vers les sources des eaux vives, ainsi la conscience assoiffée soupire vers l'absent incognito dont nul ne sait le nom. (Jankelevitch, V. 1957. Le je-ne-sais-quoi)

[25a] Il aboie des choses, des conseils sans doute, mais Solange l'entend mal et la femme a de la bave à la commissure des lèvres, sa crête de cheveux oscille sous la pluie. (Ch. De Rivoyre, Les sultans, 1964)

[25b] Catherine triompha: "chien qui aboie ne mord pas toujours. " (G. Lefebvre, La révolution française, 1963)

Une deuxième caractéristique des verbes qui indiquent des cris d'animaux est que leurs emplois transitifs correspondent souvent à des incises ou à des cas de discours direct. L'exemple [26a] est particulièrement significatif dans la mesure où on y trouve le verbe aboyer accompagné d'un SN (le complément d'objet direct est introduit par un article indéfini), alors que les paroles correspondent en fait à un discours direct.

[26a] Il ouvre la portière, jette ses jambes d'un côté, soulève son torse avec un han de colère, il est debout sur la chaussée, encore menaçant, il aboie un 
bonne chance espèce de..., claque la portière, la voiture tangue. (Ch. de Rivoyre, Les sultans, 1964)

[26b] L'une me brait : -« je ne veux pas être ton âne » et le nègre murmure : " je ne veux pas être ton nègre » en zézayant. (M. Jouhandeau, Mr. Godeau intime Véronique, 1926)

[26c] « Bah! » bêle sa chèvre qui, non loin de là, règle son compte à un jeune myosotis. (H. Bazin, Le bureau des mariages, 1951)

[26d] Au 5e, elle a eu la même réaction que le cheval normand: "je suis incrédule! " hennit-elle en montrant ses dents. Du coup, elle arrêta l'ascenseur au 6e (J. Vautrin, Billy-ze-Kick, 1974)

Le tableau ci-dessous indique le nombre de cas de discours direct ou d'incise attestés pour les verbes correspondant à des cris d'animaux :

\begin{tabular}{|l|l|l|l|}
\hline & $: \ldots \ldots *$ & $\ldots, \mathrm{V}-\mathrm{il}$ & $\begin{array}{l}\text { Total } \\
\text { transitifs }\end{array}$ \\
\hline Aboyer & 9 & 10 & 26 \\
\hline Barrir & 1 & 2 & 6 \\
\hline Bêler & 2 & 1 & 4 \\
\hline Braire & 1 & 0 & 5 \\
\hline Cacarder & 0 & 2 & 2 \\
\hline Caqueter & 2 & 1 & 3 \\
\hline Clabauder & 0 & 0 & 4 \\
\hline Coasser & 1 & 0 & 4 \\
\hline Croasser & 3 & 5 & 10 \\
\hline Feuler & 0 & 1 & 1 \\
\hline Hululer & 1 & 0 & 1 \\
\hline Jacasser & 1 & 1 & 6 \\
\hline Japper & 4 & 0 & 7 \\
\hline Meugler & 4 & 0 & 6 \\
\hline Miauler & 4 & 1 & 20 \\
\hline Mugir & 7 & 2 & 16 \\
\hline Pépier & 3 & 0 & 6 \\
\hline Piauler & 3 & 0 & 4 \\
\hline Ronronner & 4 & 0 & 5 \\
\hline
\end{tabular}

Tableau 5 : Verbes intransitifs « durs » exprimant des cris d'animaux

On voit que pour des verbes comme aboyer, croasser, mugir ou ronronner, le procédé est très productif, alors que pour d'autres verbes, tels braire ou coasser, il l'est beaucoup moins.

Parmi les verbes de parole intransitifs les plus robustes, on trouve en bonne place des verbes comme converser, causer, deviser discourir discutailler, parler qui dénotent génériquement l'activité de parole ou ses prémices (babiller). Ces verbes mettent l'accent sur l'exercice de la faculté de langage, sur l'action même d'échanger des propos indépendamment de leur signification. Le fait que les verbes comme bavarder, bavocher, blablater, parloter, papoter, potiner, pérorer soient également très bien représentés dans la catégorie des intransitifs «durs » n'a rien de surprenant dans la mesure où ils ne font qu'ajouter un trait descriptif aux verbes précédents, à savoir le fait que l'activité de parole qu'il dénote est non planifiée. Ce trait se retrouve avec bavasser, cancaner, déblatérer, sauf que ceux-ci sont marqués négativement, ils signalent que les paroles échangées sont peu importantes et/ou dépréciatives. Avec médire, la polarité négative l'emporte sur le caractère non planifié, le trait négatif est ciblé sur un tiers (particulier ou 
collectif) et les constructions avec complément indirect en de prédominent (78\%). Le même phénomène se retrouve avec déblatérer et jaser, qui font partie des intransitifs "durs ", ainsi qu'avec baver et dégoiser qui se construisent eux aussi avec la préposition sur mais sont plus "mous». Ce glissement vers la transitivité est confirmé par le fait qu'un grand nombre de verbes de sens proche comme débiner, dénigrer, calomnier, décrier, diffamer sont transitifs ${ }^{16}$. Quoiqu'il soit difficile de généraliser, il semble bien que les verbes de parole intransitifs les plus « durs » ont en commun de focaliser sur un aspect de la production de paroles, soit sur une caractéristique formelle des propos (verbes dénotant un genre, un registre, un dialecte, un type de cri, etc.), soit sur leur caractère peu contrôlé, l'évocation du contenu des paroles tendant par contre à favoriser le passage à la transitivité.

\subsection{Les verbes intransitifs « mous »}

Cette catégorie regroupe les verbes qui s'avèrent, après recherche sur corpus, au moins aussi transitifs qu'intransitifs. Contrairement aux intransitifs "durs», les intransitifs «mous » sont plus disparates du point de vue sémantique. Si on y retrouve plusieurs des catégories mentionnées plus haut, elles sont toutefois moins bien représentées. Ainsi on peut distinguer les catégories suivantes ${ }^{17}$ :

- des verbes qui renvoient à la forme de l'élocution: bafouiller, bégayer, chevroter, éructer grasseyer, nasiller, tonitruer, vitupérer;

- des verbes de cris d'animaux : beugler, glapir, grognasser;

- certains verbes qui renvoient à l'état mental ou à l'attitude psychologique du locuteur: fulminer, baver, maugréer, rouscailler, pester, tonitruer, rager, convenir, médire, criticailler;

- des verbes dont le contenu correspond à un certain genre littéraire ou à un type particulier de discours : argumenter, baratiner, débattre, disputer, chicaner, chinoiser, pétitionner.

Voici quelques exemples :

[27a] L'armée de Condé frayant la voie aux troupes étrangères, on rétablirait l'ancien régime: les émigrés fulminaient les plus terribles menaces contre leurs compatriotes et parlaient avec mépris de leur souverain qui supportait docilement le joug de l'assemblée. (G. Lefebvre, La révolution française, 1963) [27b] Ses messages en morse, saccadés, déphasés, qui coupent la narration tout à trac comme s'ils étaient captés d'une autre planète, bégayent déjà des nouvelles de la contrée où va s'éveiller Rimbaud. (J. Gracq, Préférences, 1961) [27c] Il m'argumentait qu'il fallait des choses de ce style pour avoir quelques chances d'allécher une star. (A. Boudard, Les enfants de chœur, 1982) [27d] Secouant son chapeau melon, un homme bondit sur la chaussée pour mieux beugler son enthousiasme. (Y. Gibeau, Allons z'enfants, 1952)

Pour ce qui est des 3 verbes de cris d'animaux, la même observation s'impose que dans le cas des verbes intransitifs « durs » : ils se transitivisent lorsqu'ils sont employés en incise ou en discours direct. Ainsi dans le cas de beugler, les emplois transitifs correspondent à des discours directs ( 19 cas) ou des incises (14 cas), soit 33 cas contre 18 cas de SN et 1 cas de complétive que $P$ :

[28a] Lamure ne l'entendit pas. Il beuglait trop haut: «- Gauche, droite! Gauche, droite!... Gauche!... Gauche!... » (H. Bazin, Le bureau des mariages, 1951)

[28b] « A l'aventure ! » beugla Antoine. (R. Fallet, Le triporteur, 1951) 
[28c] La morèna rassemble les outres flasques de sa poitrine entre ses gros bras trop courts, se penche, relève la tête, beugle au ciel son désespoir. (J.-P. Chabrol, Je t'aimerai sans vergogne, 1967)

De même pour glapir : il y a 35 cas d'incise, 25 cas de discours direct, 12 cas de SN et 2 de complétive :

[29a] «C'est une tribu, ce n'est pas une ville», glapit encore Suzanne. (H. Bazin, La mort du petit cheval, 1950)

[29b] Par-dessus le brouhaha des voix mêlées, celle de la fille glapit, suraiguë : «Vingt sous ! C'est vingt sous !... Poussez pas comme ça, voyons ! Poussez pas, ou j'ferme la porte !» (M. Genevoix, Ceux de 14, 1950)

[29c] ... il se mit à glapir d'une voix affreusement enjouée des fragments de son répertoire de caf'conc', dont le refrain intéressant et quelque peu énigmatique est resté dans ma mémoire ... (R. Gary, La promesse de l'aube, 1960)

L'exemple suivant où discours direct et complément d'objet direct fusionnent dans le même complément est particulièrement révélateur pour la question de la transitivité discutée ici. On voit en effet in vivo comment le discours direct constitue une " étape-clé " dans le passage de l'intransitivité à la transitivité :

[30] Mammouth, à nouveau dirigé sur l'obstacle, décrivit un soleil et se réceptionna sur le menton en beuglant un second «Crénom! » (R. Fallet, Le triporteur, 1951)

Quant à grognasser, il est suivi soit d'un SN [31a] soit d'une citation [31b] mais ce sont les deux seuls exemples attestés dans Frantext:

[31a] Importe comment, je me cherchais pas de musique... mais là, bon gré, mal gré, il me la faut!... je dirais même, une mélodie...voyez-moi ça! pas instruit ni doué forcé de me grognasser des bribes... autre chose! mes cannes!...perdu les deux dans cette idiote explosion... que tout s'est abattu sur nous, enfin la façade... je crois, je suis pas sûr... (L-F. Céline, Rigodon, 1961) [31b] ... passée de main en main, ramassé un drapeau rouge tombé derrière les grilles de Cluny, dansé la farandole autour d'un car incendié, entendu les syndicalistes grognasser devant des gars et des filles fous de joie et d'espoir : «C'est de Gaulle qui a poussé à faire des enfants ! Voilà ce que ça donne ! ... » (J-P. Chabrol, La folie des miens, 1977)

Certains verbes qui n'appartiennent pas à la catégorie des verbes de cris d'animaux, se transitivisent également au moyen de l'incise ou du discours direct, mais comme le montre le tableau suivant, la distribution est loin d'être homogène : 


\begin{tabular}{|l|l|l|l|}
\hline & $:$ «... & $\ldots ., \mathbf{V}_{\text {-il }} \mathbf{1}^{\mathbf{1}}$ & Total transitifs \\
\hline Argumenter & 3 & 4 & 13 \\
\hline Bafouiller & 18 & 22 & 68 \\
\hline Baratiner & 1 & 0 & 20 \\
\hline Baver & 0 & 3 & 15 \\
\hline Bégaver & 20 & 19 & 57 \\
\hline Chicaner & 0 & 0 & 91 \\
\hline Chinoiser & 0 & 0 & 2 \\
\hline Convenir & 0 & 0 & 125 \\
\hline Criticailler & 0 & 0 & 1 \\
\hline Débattre & 0 & 0 & 24 \\
\hline Dégoiser & 0 & 0 & 16 \\
\hline Disputer & 0 & 0 & 62 \\
\hline Eructer & 3 & 5 & 18 \\
\hline Fulminer & 5 & 11 & 21 \\
\hline Grasseyer & 4 & 14 & 24 \\
\hline Discuter & 0 & 0 & 106 \\
\hline
\end{tabular}

Tableau 6 : Cas d'incise et de discours direct (verbes ne dénotant pas des cris d'animaux)

direct précisent souvent la façon dont sont proférées les paroles : il s'agit de bafouiller, bégayer, grasseyer et fulminer (ce dernier caractérisant en plus l'attitude du locuteur). On pourrait proposer une analyse de ces verbes, ainsi que ceux des cris d'animaux, par fusion (Gross, 1981) :

[32a] Paul (bafouille + glapit) : $X$

[32b] X, (bafouilla-t-il + glapit-il)

[32c] Paul dit en (bafouillant + glapissant) : $X$

[32d] X, dit-il en (bafouillant + glapissant)

Dans les cas où on observe un taux élevé d'emplois transitifs sans que la transitivité soit due à l'incise ou au discours direct, il ne s'agit pas de verbes occasionnellement transitifs, comme ceux dont on vient de parler, mais de verbes dont la valence prévoit une structure transitive et intransitive à la fois. C'est le cas, par exemple de chicaner, de convenir, ou de discuter. Alors que chicaner sélectionne plus souvent un SN de type [+hum] (66 cas) qu'un SN inanimé (25 cas), convenir se construit presque toujours avec une complétive (ou infinitive) : 118 cas contre 7 cas de $\mathrm{SN}^{18}$. Discuter enfin sélectionne le plus souvent un SN inanimé (104 cas contre 1 cas de SN [+hum] et un cas d'interrogation indirecte) :

[33a] La droite comptait obliger le gouvernement à la paix en lui coupant les vivres; la gauche le chicanait pour diminuer son autorité. (G. Lefebvre, La révolution française, 1963)

[33b] Encore bien loin de cet âge où mutuellement l'homme et la femme se trouvent trop près de l'avenir pour perdre du temps et se chicaner leurs jouissances, elle en était, sans doute, non pas à son premier amour, mais à ses premiers plaisirs. (H. de Balzac, Histoire des treize, 1835) ${ }^{19}$

[34a] Il était convenu qu'il l'enverrait prendre à Trye, qu'il l'hébergerait au temple quand il traverserait Paris. (J. Guéhenno, Jean-Jacques, 1952)

[34b] Je te comprends, et je sais que tu me comprends, comme tu comprends toi-même que tu es compris ; autrement dit, j'ai conscience de ta conscience, qui est à son tour consciente de la mienne; tout cela, naturellement, sans le dire, car rien n'a jamais été convenu ni expressément stipulé. (V. Jankelevitch, Le je-ne-sais-quoi, 1957) 
[35a] Je m'excuse, mesdames et messieurs, de ne pouvoir entrer dans le détail de tous les articles du décret, et de ne pouvoir les discuter tous. (C. Pineau, S.N.C.F. et transports français, 1950.)

[35b] On discuta encore si l'acte de donation pour être valable devait être passé en Hollande ou en France. (J. Guéhenno, Jean-Jacques, 1952)

Étant donné que convenir est bien plus souvent attesté dans le corpus comme transitif (77\%) que comme intransitif ( $23 \%$ ), on pourrait le considérer comme essentiellement transitif, et occasionnellement intransitif (Larjavaara, 2000). Il en va de même des verbes baratiner et disputer, dont les pourcentages sont encore plus parlants : $13 \%$ de Vi et $87 \%$ de $\mathrm{Vt}, 12 \%$ de $\mathrm{Vi}$ et $88 \%$ de $\mathrm{Vt}$, respectivement. Le verbe baratiner prend comme c.o.d. un SN de type [+hum] dans 15 cas sur 20, mais on le trouve avec un SN de type inanimé dans 3 cas, avec une complétive que $P$ dans 2 cas et une seule fois en discours direct :

[36a] Il pouvait me baratiner, j'avais quelques idées sur la question. (A. Boudard, La cerise, 1963)

[36b] Quand j'allais chez lui des fois d'temps en temps J'lui roulais ses clopes avec son tabac gris Pi j'restais des heures avec des yeux tout grands à l'écouter m'baratiner sa vie. (Renaud, Mistral gagnant, 1985)

[36c] Je lui ai baratiné que j'ai rempli un dossier au bureau de chômage. Ah ! ah !... Chien de menteur que je suis ! (M. Charef, Le thé au harem, 1983)

Même si certains de ces exemples sont marqués du point de vue du registre, il est significatif qu'ils apparaissent dans la construction formelle prototypique des verbes de parole, à savoir N0 V (N1 + que P) + à N2. Dans le cas de disputer, la majorité des c.o.d. correspondent à des $\mathrm{SN}$ de type inanimé (50 cas), alors que l'objet direct de type [+hum ], le plus souvent de type réfléchi ou réciproque, apparaît dans 14 cas [37b]. Dans la plupart des emplois où le c.o.d. est inanimé, un c.o.i. est présent également (30 cas contre 21 cas sans datif) comme dans [37a] :

[37a] Quant aux choses sérieuses, Mme Du Barry disputait le pouvoir à M. De Choiseul. C'est dans ce monde-là qu'il tombait avec ses rides et ses idées noires. La police ne l'inquiéta pas. (J. Guéhenno, Jean-Jacques, 1952)

[37b] Après cela, nous pouvons continuer, pendant des années encore, à nous disputer sur la manière dont a pu surgir cet organisme énorme. (Teilhard de Chardin, Le phénomène humain, 1955)

\section{Conclusions}

À la question posée au début de cet article, à savoir s'il existe un rapport entre transitivité syntaxique et sémantique verbale, la réponse est positive dans le cas des verbes de parole: si une première étude (Charolles, Lamiroy, 2001 ; Lamiroy 2005) avait montré qu'il existe un contraste quantitatif important entre verbes de parole transitifs (env. 350 V) et intransitifs (env. $150 \mathrm{~V}$ ), l'étude de corpus présentée ici a révélé que $20 \%$ des 142 verbes étudiés, étiquetés comme intransitifs par le PRe (1997) et attestés dans Frantext, sont transitifs dans au moins $40 \%$ ou plus de leurs emplois. Autrement dit, l'hypothèse initiale est non seulement confirmée, mais renforcée: les verbes qui impliquent conceptuellement, dans leur grille sémantique, un objet, comme c'est le cas des verbes de parole, entrent très souvent dans des structures transitives. Au vu de ce résultat, on peut s'étonner de l'information donnée dans les dictionnaires, qui, on l'a vu, s'est souvent avérée inexacte, ou en tout cas, trop peu nuancée. 
51 Toutefois, notre étude de corpus montre également qu'il existe un noyau irréductible de verbes étiquetés dans les dictionnaires comme intransitifs qui s'emploient effectivement sans objet : en effet, $22,5 \%$ des verbes étudiés n'apparaissent jamais en emploi transitif (ils sont donc à $100 \%$ intransitifs). Si on ajoute les verbes qui dans 80 à $100 \%$ de leurs emplois sont attestés comme intransitifs, on atteint $64 \%$ des verbes. Il semble donc justifié de parler d'un noyau « dur » de verbes de parole intransitifs.

Le rapport entre (in)transitivité et catégories sémantiques se confirme également après étude de corpus. En particulier, les verbes qui spécifient la façon particulière dont les paroles sont proférées sont essentiellement intransitifs. Cela est vrai pour des verbes comme babiller et, de façon très manifeste, pour les verbes indiquant des cris d'animaux. Nous avons en effet observé que parmi ceux-ci, $70 \%$ font partie de la catégorie des verbes intransitifs " durs ", c'est-à-dire qu'ils sont intransitifs entre 80 et 100\% des cas examinés. Or nous avons observé une régularité importante pour ce type de verbes: ils se prêtent facilement à un emploi transitif dès qu'ils ont un sens métaphorique. Il faut noter par ailleurs qu'il existe des exceptions lexicales : certains verbes tels bafouiller et bégayer qui renvoient bien à la manière de parler sont employés transitivement dans plus de la moitié des emplois attestés. De même, les verbes glapir ( $23 \% \mathrm{Vi}-77 \% \mathrm{Vt}$ ) et beugler (32\% Vi - $68 \%$ $\mathrm{Vt})$, pourtant des verbes de cris d'animaux sont hautement transitifs, le verbe chevroter se répartissant de façon égale entre les deux (50\% Vi - 50\% Vt).

Une autre catégorie sémantique clairement représentée par des emplois intransitifs est celle qui renvoie au caractère non planifié des paroles. Comme leur objet n'est pas profilé, les verbes ne s'accompagnent pas d'un c.o.d. Ainsi, les verbes bavarder, blablater, blatérer, bavocher et bléser sont à $100 \%$ intransitifs. Dans le cas de causer, qui est un verbe générique, le pourcentage des cas intransitifs atteint $97 \%$.

Quant à la catégorie concernant l'état mental ou psychologique du locuteur, les chiffres obtenus montrent qu'il faut distinguer deux sous-types : si les verbes renvoyant à l'état mental du locuteur sont essentiellement intransitifs (divaguer et digresser sont à $100 \%$ intransitif, délirer à 98\%), ceux qui indiquent plutôt un sentiment ou une attitude psychologique du locuteur par rapport à l'interlocuteur, sont moins clairement intransitifs (fulminer n'est intransitif que dans $58 \%$ pour des cas, éructer dans $50 \%$ des cas).

Nous avons constaté que les catégories sémantiques discernées conviennent mieux aux verbes intransitifs "durs» qu'aux intransitifs «mous». La distinction entre verbes « durs » et « mous » nous parait intéressante dans la mesure où nous avons pu observer en effet qu'il y a des verbes essentiellement intransitifs qui ne sont qu'occasionnellement transitifs alors qu'il y en a d'autres qui sont vraiment à la fois transitifs et intransitifs, du moins dans notre corpus. Ainsi, certains verbes apparaissent de façon surprenante parmi les verbes qui s'emploient autant (ou presque) de façon transitive qu'intransitive : nous avons vu plus haut le cas de bégayer ou bafouiller, mais il en va de même des verbes chicaner (env. 60\% d'emplois transitifs contre env. $40 \%$ d'emplois intransitifs), baratiner (presque $90 \%$ d'emplois transitifs) ou jurer (71\% d'emplois transitifs contre $19 \%$ d'emplois intransitifs).

56 Une autre hypothèse qui se voit confirmée est celle de la relation entre atélicité et intransitivité : des verbes comme criailler, écrivailler, écrivasser, discutailler font partie des intransitifs « durs ».

57 Enfin, nous avons insisté sur l'intérêt de l'incise et du discours direct dans cette problématique, parce qu'ils représentent à notre avis les cas extrêmes d'une hiérarchie 
allant de verbes peu transitifs à des verbes «très » transitifs. Ce qui ressort de notre étude est que la catégorie pour laquelle ce type de procédé est le plus productif correspond à celle qui spécifie la forme du message, qu'il s'agisse de cris d'animaux ou d'autres verbes ( bégayer, bafouiller). Ces verbes sont virtuellement transitifs parce qu'ils permettent une extension par « fusion » (Gross, 1981).

Notons toutefois, pour finir, que cette étude devrait être complétée par l'examen détaillé des verbes de parole transitifs, notamment de ceux qui spécifient la manière, comme par exemple murmurer pour voir si, à l'inverse de ceux que nous avons étudiés ici, ils se prêtent à des emplois intransitifs.

\section{BIBLIOGRAPHIE}

BOONS, J-P., GUILLET, A., LECLÈRE, C. 1976. La structure des phrases simples en français. Constructions intransitives. Genève : Droz.

CHAROLLES, M., LAMIROY, B. 2001. Zézayer, dire ou parler : les verbes de parole ou qu'est-ce qu'un verbe (in)transitif? XXe Colloque International de Lexique et Grammaire Comparés. Londres. Poster. CHUQUET, J. (éd.) 2004. Verbes de parole, de pensée, de perception : études syntaxiques et sémantiques. Rennes : Presses Universitaires de Rennes.

CRISTOFARO, S. 2003. Subordination. Oxford : Oxford University Press.

DIK, S., HENGEVELD, K. 1991. The Hierarchical Structure of the Clause and the Typology of Perception-Verb Complements. Linguistics 29 : 231-259.

FRANÇOIS, J. 1999. Les caractères aspectuels et participatifs des prédications verbales et la transitivité. Bulletin de la Société Linguistique de Paris XCIV, fasc. 1 : 139-184.

GIIRY-SCHNEIDER, J. 1981. Les compléments nominaux du verbe 'dire'. Langages 63 : 75-95.

GIVON, T. 1984. Syntax. A Functional-Typological Introduction. Amsterdam : Benjamins.

GROSS, M. 1975. Méthodes en Syntaxe. Paris : Hermann.

GROSS, M. 1981. Les bases empiriques de la notion de prédicat sémantique. Langages 63 : 7-52.

GUILLET, A., LECLÈRE, C. 1992. La structure des phrases simples en français. Constructions transitives locatives. Genève : Droz.

HERSLUND, M. 2001. Romance Transitivity. K. DAVIDSE, B. LAMIroy (éds) Case and Grammatical Relations. The Nominative-Accusative. Amsterdam : Benjamins. 323-349 (sous presse). HOPPER, THOMSON, S. 1985. Transitivity in Grammar and Discourse. Language 56, 2 : 251-297. JACKIEWICZ, A. 2005. Transmission dialogique des paroles d'autrui. Verbum XXVII, 3 : 265-292. JAMROZIK, E. 1992. La syntaxe et la sémantique des verbes de parole français. Varsovie: Wydawnictwa Uniwersytetu.

KÜBLER, N. 1994. Etude des objets nominaux de verbes de parole anglais, comparaison avec le français. Langages $28: 115,76-101$. 
LAMIROY, B. 2005. From syntax to semantics : the case of intransitive speech verbs. I. BARON, H. KORZEN, I. KORZEN, H. MÜLLER, H. NøLKE (eds). Grammatica. Festschrift in Honour of Michael Herslund. Bern : P. Lang. 279-295.

LANGACKER, R. 1987. Foundations of Cognitive Grammar. Stanford : Stanford University Press.

LarjaVAara, M. 2000. Présence ou absence de l'objet. Helsinki : Academia Scientiarium Fennica.

LAZARD, G. 1994. L'actance. Paris : PUF.

LEVIN, B. 1993. English Verb Classes and Alternations. Chicago : Chicago University Press.

LYONS, J. 1977. Natural Language and Universal Grammar. Cambridge : CUP.

RIEGEL, M., PELLAT, J.-C., RIOUL, R. 1994. Grammaire méthodique du français. Paris : PUF.

SARDA, L. 2000. L'expression du déplacement dans la construction transitive directe. Syntaxe et Sémantique 2 :121-137.

SEARLE J., 1979, 1982. Sens et expression. Paris : Minuit. (Chap.I)

STASSEN, L. 1997. Intransitive Predication. Oxford : Clarendon Press.

TALMY, L. 1985. Lexicalisation patterns : semantic structure in lexical forms. T. SHOPEN (éd.)

Language Typology and Syntactic Description. Cambridge : CUP. 57-149.

WIERZBICKA, A. 1987. English Speech Act verbs. A semantic dictionary. Sydney : Academic Press.

\section{NOTES}

1. Le fait même qu'on parle de verbes de parole (cf. Jamrozik 1992) ou verbes d'énonciation (Riegel, 1994 :221), de speech verbs (Wierzbicka, 1987) ou utterance predicates (Cristofaro, 2003), tout comme on parle de verbes de mouvement, de verbes de sentiment, de verbes de volonté, etc. suggère qu'ils constituent des ensembles relativement homogènes du point de vue sémantique.

2. Il y aurait quelque 120 verbes locatifs transitifs, tels traverser (la rue) ou atteindre (le sommet) contre plus de 1400 verbes intransitifs (du type aller, passer (par là) recensés dans les tables du LADL (Sarda, $2000: 122$, note 3).

3. Les notations utilisées sont empruntées à Gross (1975). Le signe + a une valeur disjonctive, E renvoie à un élément facultatif (cette position peut donc être vide) et les () signalent la forme (directe vs indirecte) sous laquelle le complément peut apparaître.

4. Dans un cas comme délirer, il y a production de paroles sans qu'il y ait l'intention de communiquer.

5. Riegel (1994 :221) fait une remarque analogue (sans nommer explicitement le principe) quand il dit des verbes intransitifs que «leur sens globalise un procès en intégrant les spécifications qui pourraient être exprimées par des compléments ».

6. Nous adoptons les définitions de Comrie (1976). Si le concept d'(a)télicité est plutôt lié à l' Aktionsart du verbe, celui d'(im)perfectivité concerne plutôt l'aspect morphosyntaxique, mais les deux sont évidemment liés : "Thus the situation described by make a chair has built into it a terminal point, namely that point at which the chair is complete, when it automatically terminates; the situation described by sing has no such terminal point, and can be protracted indefinitely or broken off at any point. Situations like that described by make a chair are called telic, those like that described by sing atelic " (1976: 44). "Another way of explaining the difference between perfective and imperfective meaning is to say that the perfective looks at the situation from outside, without necessarily distinguishing any of the internal structure of the 
situation, whereas the imperfective looks at the situation from inside, as such is crucially concerned with the internal structure of the situation » (1976:4).

7. Ce classement est intuitif, et comme tout classement sémantique, en partie arbitraire. Les catégories retenues, même si elles ne couvrent pas la totalité des cas, permettent toutefois de catégoriser la plupart des verbes de parole retenus.

8. Pour une classification sémantique des verbes introducteurs de discours rapporté et des modalités (souvent exprimées par des expressions prépositionnelles) les accompagnant, cf. (Jackiewicz, 2005).

9. Deux autres aspects du "contenu" ont été pris en compte, à savoir la conformité à un certain genre, p.ex. pindariser, rimailler et l'évaluation par rapport à la norme, p.ex. mentir, parjurer. Il est évident que certains verbes pourraient être classés sous plus d'une rubrique et que le classement a donc une valeur relative.

10. Giry-Schneider (1994) fait remarquer que l'introduction d'un complément datif peut transformer un verbe en verbe de parole, p.ex. Jean nous a déchiffré que le train arrivait à 8 heures.

11. Ce travail a été effectué dans des mémoires de maîtrise par trois étudiants de l'université de Leuven : Nadia Goffings, Annelies Soenen et Marie Van Gorp que nous remercions pour leur aide.

12. Certains verbes qui se prêtent à des emplois métaphoriques avec un sujet humain comme tonner n'ont pas été pris en compte non plus, par ex. «Le curé tonne contre la Bovary et défend à ses paroissiennes de me lire. Vous allez me trouver bête mais ç'a été pour moi une grande joie de vanité. » ( Flaubert, lettre à Jules Duplan).

13. Les verbes dénotant des cris d'animaux sont en caractère droit.

14. Le verbe criticailler fait exception ici puisqu'il n'est jamais attesté comme verbe intransitif; mais il faut tenir compte du fait que les chiffres sont peu représentatifs: il s'agit d'un seul exemple.

15. Liste des verbes dénotant des cris d'animaux: aboyer ; barrir ; bêler ; beugler ; braire ; bramer ; cacarder ; caqueter ; chevroter ; clabauder ; coasser ; croasser ; feuler ; gazouiller ; glapir ; glousser ; grognasser ; hululer ; jacasser ; japper ; meugler ; miauler ; mugir ; pépier ; piauler ; ronronner ; roucouler ; striduler.

16. Dans (Charolles, Lamiroy, 2001) ces verbes sont répertoriés sous la catégorie verbes dénotant des actes de langage expressif négatif (voir classement sémantique des verbes de parole transitifs).

17. Comme dans tout classement, certains cas résiduels sont difficiles à classer : le verbe convenir en est un exemple.

18. Pour convenir, il faut noter un très grand nombre de cas passifs: dans plus d'un cas on peut se demander si on a affaire à un participe passé passif ou à un adjectif verbal. Cette question dépasse toutefois le cadre de cet article.

19. D’après le Petit Robert, cet emploi de chicaner avec SN inanimé serait vieilli.

\section{RÉSUMÉS}

Le but de l'article est d'examiner la relation entre un aspect de la syntaxe verbale (la transitivité) et les caractéristiques sémantiques d'un ensemble de verbes, celui des verbes de parole. L'hypothèse fondamentale est que ces verbes impliquent par nature un objet (les paroles produites) et qu'ils sont donc naturellement transitifs, ce qui se vérifie pour la grande majorité 
des verbes. Comme la question se pose de savoir pourquoi certains verbes de parole sont tout de même intransitifs (p. ex. bégayer, zozoter) se pose, une deuxième hypothèse est explorée qui prédit un rapport inverse entre transitivité syntaxique et spécification sémantique : si le sémantisme d'un verbe est hautement spécifié, le verbe serait saturé lexicalement, et syntaxiquement intransitif. Une place à part est accordée aux verbes de cris d'animaux, qui tout en étant intransitifs au départ se prêtent très facilement à des emplois transitifs, en particulier dans le discours rapporté.

The focus of the paper is the relationship between the syntax and the semantics of speech verbs. We hypothesize that speech verbs are naturally transitive because they refer to an activity which normally entails an object, viz. the produced speech. Most speech verbs are in effect transitive. However, to account for those verbs which are intransitive (e.g. bégayer, zozoter), we explore a second hypothesis based on a principle of semantic saturation: if the semantics of a verb is highly specific, then the verb is lexically saturated, and hence, syntactically intransitive. Verbs that indicate animal cries occupy a special position in that they are basically intransitive but lend themselves to transitive uses, especially in reported speech.

INDEX

Keywords : transitive verb, speech verb, intransitive verb

Mots-clés : verbe de parole, verbe transitif, verbe intransitif

\section{AUTEURS}

\section{BÉATRICE LAMIROY}

Université de Leuven Beatrice.Lamiroy@arts.kuleuven.be

\section{MICHEL CHAROLLES}

Université de Paris 3 - UMR LATTICE Michel.charolles@ens.fr 